\title{
The role of cholesterol-sphingomyelin membrane nanodomains in the stability of intercellular membrane nanotubes
}

This article was published in the following Dove Press journal:

International Journal of Nanomedicine

6 April 2012

Number of times this article has been viewed

\author{
Maruša Lokar ${ }^{1, *}$ \\ Doron Kabaso ${ }^{1,2, *}$ \\ Nataša Resnik ${ }^{3}$ \\ Kristina Sepčić ${ }^{5}$ \\ Veronika Kralj-Iglič 4,6 \\ Peter Veranič ${ }^{3}$ \\ Robert Zorec ${ }^{2}$ \\ Aleš Iglič́ ${ }^{1,6}$
}

'Laboratory of Biophysics, Faculty of Electrical Engineering, ${ }^{2}$ Laboratory of Neuroendocrinology-Molecular Cell Physiology, Faculty of Medicine, ${ }^{3}$ Institute of Cell Biology, Faculty of Medicine, ${ }^{4}$ Faculty of Health Sciences, ${ }^{5}$ Department of Biology, Biotechnical Faculty, ${ }^{6}$ Laboratory of Clinical Biophysics, Department of Orthopedic Surgery, Faculty of Medicine, University of Ljubljana, Ljubljana, Slovenia

*These authors equally share first authorship
Correspondence: Doron Kabaso Laboratory of Biophysics, Faculty of Electrical Engineering, University of Ljubljana, 1000 Ljubljana, Slovenia Tel +386 I 4768825

Fax +386 I 4768850

Email doron.kabaso@fe.uni-lj.si

\begin{abstract}
Intercellular membrane nanotubes (ICNs) are highly curved tubular structures that connect neighboring cells. The stability of these structures depends on the inner cytoskeleton and the cell membrane composition. Yet, due to the difficulty in the extraction of ICNs, the cell membrane composition remains elusive. In the present study, a raft marker, ostreolysin, revealed the enrichment of cholesterol-sphingomyelin membrane nanodomains along ICNs in a T24 (malignant) urothelial cancer cell line. Cholesterol depletion, due to the addition of methyl- $\beta$ cyclodextrin, caused the dispersion of cholesterol-sphingomyelin membrane nanodomains and the retraction of ICNs. The depletion of cholesterol also led to cytoskeleton reorganization and to formation of actin stress fibers. Live cell imaging data revealed the possible functional coupling between the change from polygonal to spherical shape, cell separation, and the disconnection of ICNs. The ICN was modeled as an axisymmetric tubular structure, enabling us to investigate the effects of cholesterol content on the ICN curvature. The removal of cholesterol was predicted to reduce the positive spontaneous curvature of the remaining membrane components, increasing their curvature mismatch with the tube curvature. The mechanisms by which the increased curvature mismatch could contribute to the disconnection of ICNs are discussed.
\end{abstract}

Keywords: cyclodextrins, T24 urothelial cancer cell line, intercellular membrane nanotubes, cholesterol-sphingomyelin membrane nanodomains

\section{Introduction}

Intercellular membrane nanotubes (ICNs), as a novel intercellular communication mechanism, are extensively studied both functionally and structurally. ${ }^{1-7}$ Previously, it has been shown that ICNs are stabilized not only by the inner cytoskeleton but also by the membrane composition. ${ }^{8-10}$ Yet, due to their fragility, it is impossible to separate the ICNs from the rest of the cell, and therefore their membrane composition remains unknown. To shed light on the ICN membrane composition, it is possible to use membrane raft markers, for the binding of different lipids, proteins, and their complexes. ${ }^{11-16}$ In the present study, the addition of a raft marker, ostreolysin (Oly), revealed the presence of cholesterolsphingomyelin membrane nanodomains throughout the plasma membrane and ICNs. The role of cholesterol-sphingomyelin nanodomains in the stability of ICNs was explored using a cholesterol depletion agent and a cholesterol-free growth medium.

Oly belongs to a novel protein family called aegerolysins (PF06355; IPR 009413), which are known for their cytolytic properties. ${ }^{17,18}$ The binding of Oly evokes the formation of distinctive pores that induce cell lysis. ${ }^{19}$ Fluorescent mutants devoid of lytic activity, or fluorescent antibody-labeled proteins at their sublytic concentrations, have been used for the structural investigation of biological membranes. ${ }^{11-16}$ In particular, 
it has been revealed that Oly interacts specifically with cholesterol-sphingomyelin membrane nanodomains, ${ }^{19-21}$ which do not colocalize with caveolin and cholera toxin B membrane nanodomains. ${ }^{11}$ Oly differs from other raft markers, since it can sense the interaction between two lipids (ie, cholesterol and sphingomyelin), and does not bind to any lipid in its pure form.

While cholesterol-enriched membrane nanodomains have been found to be important for different biological functions, such as exocytosis and endocytosis, signal transduction, pathogen entry, and attachment of various ligands, ${ }^{22-24}$ their contribution to the stability of highly curved membrane regions (eg, ICNs) are not fully understood. A cholesterol molecule has a small hydrophilic headgroup and a relatively short hydrophobic tail, which enables it to fill gaps in highly curved cell membrane regions. ${ }^{25,26}$ The stability of a highly curved ICN might depend on the relative concentration of cholesterol. In mixed membrane models, the addition of cholesterol facilitates the formation of an intermediate phase called liquid ordered phase, ${ }^{27,28}$ exhibiting more rigidity than the surrounding liquid disordered nanodomains. ${ }^{29}$ Therefore, it is possible that the stability of the ICN may depend on the distribution of liquid ordered membrane nanodomains enriched in cholesterol. In the literature, liquid ordered membrane nanodomains were previously denoted as membrane rafts. ${ }^{22,23}$ Membrane rafts are defined as small (10-300 nm) in diameter, heterogeneous, and highly dynamic domains. Upon stimulation, small rafts can be stabilized to form larger platforms through protein-protein and protein-lipid interactions.

Cyclodextrins (CDs) are a family of cyclic compounds made up of oligosaccharides that absorb cholesterol from the cell membrane. The inner core of the CD toroidal structure is much less hydrophilic than the outer surface, which facilitates the absorption of cholesterol from the cell membrane. ${ }^{30,31}$ On the other hand, the release of phospholipids from cell membranes under exposure to $\mathrm{CD}$ is only minimal. For example, less than $5 \%$ of the initial cellassociated choline-labeled phospholipids was released after 1-hour exposure with $1 \mathrm{mM} \mathrm{CD}$ in various Chinese hamster ovary cells and, at most, $2 \%$ of cellular phospholipids was released after 5-hour incubation with $5 \mathrm{mM} \mathrm{CD}$ from L-cells. ${ }^{32,33}$ Besides their effect on membrane composition, other studies investigated the effect of CDs on cellular communication and cell shaping, demonstrating a decrease in the frequency of endocytosis and in the numbers of membrane invaginations. ${ }^{34-36}$

Herein, the effects of cholesterol depletion on the mesenchymal shape of a T24 cell and on the density of ICNs are reported. It is revealed that either methyl- $\beta$-cyclodextrin $(\mathrm{m} \beta \mathrm{CD})$ treatment or the growth in cholesterol-free medium cause a significant reduction in the density of ICNs. The dynamic morphological alterations are explored using live cell imaging and confocal microscopy. A computational model was constructed to investigate the underlying mechanisms accounting for the instability of tubular structures following the application of a cholesterol depletion agent.

\section{Materials and methods Urothelial cell culture}

Urothelial cell line T24 was cultured in a 1:1 mixture of advanced Gibco ${ }^{\circledR}$ Dulbecco's modified Eagle's medium (Invitrogen Life Technologies, Carlsbad, CA) and Ham's F-12 (Sigma-Aldrich Corporation, St Louis, MO), supplemented with Gibco 10\% fetal bovine serum, $5 \mu \mathrm{g} / \mathrm{mL}$ insulin, $5 \mu \mathrm{g} /$ $\mathrm{mL}$ transferrin, $100 \mathrm{mg} / \mathrm{mL}$ hydrocortisone, and $5 \mathrm{ng} / \mathrm{mL}$ selenite (Invitrogen), as well as $1800 \mathrm{U} / \mathrm{mL}$ cristacyclin (Pliva, Zagreb, Croatia) and $0.222 \mathrm{mg} / \mathrm{mL}$ streptomycin sulfate (Fatol Arzneilmittel GmbH, Schiffweiler, Germany). Cells were incubated at $37^{\circ} \mathrm{C}$ in a humidified incubator (Heracell, Heraeus, Germany) in an atmosphere of 5\% carbon dioxide. One day prior to experiment, cells were seeded onto sterile glass coverslips (Brand GmbH, Wertheim, Germany) at approximately $70 \%-80 \%$ confluence and incubated overnight at $37^{\circ} \mathrm{C}$. In some experiments, cells were grown in a medium with lipoprotein poor serum (LPPS medium) purchased from Thermo Scientific HyClone (South Logan, UT).

\section{$\mathrm{m} \beta C D$}

The growth medium of overnight cultures was replaced with cholesterol free media (LPPS) with $1 \mathrm{mM}, 2 \mathrm{mM}$, $4 \mathrm{mM}, 6 \mathrm{mM}, 8 \mathrm{mM}, 10 \mathrm{mM}, 12 \mathrm{mM}, 16 \mathrm{mM}$, or $20 \mathrm{mM}$ $\mathrm{m} \beta C D$ (Sigma-Aldrich). Cells were incubated in $\mathrm{m} \beta C D$ for 2 hours or 4 hours, depending on the experiment. Afterwards, incubation medium was removed, cells were fixed in $2 \%$ paraformaldehyde (Merck, Hohenbrunn, Germany; $37^{\circ} \mathrm{C}$ ) for 20 minutes, washed twice in phosphate-buffered saline (PBS; Sigma-Aldrich $37^{\circ} \mathrm{C}$ ) for 15 minutes, and then analyzed (see below).

\section{Quantification of cellular cholesterol}

Lipids from cell homogenates were extracted according to the method of Bligh and Dyer. ${ }^{37}$ Briefly, for each $200 \mu \mathrm{l}$ of volume, a 1:2 mixture of chloroform:methanol (volume/ volume) was added. The chloroform layer was dried under a stream of nitrogen gas and lipids redissolved in isopropyl alcohol. The total cholesterol content was assayed using the 
Cholesterol Reagent kit (Thermo Fisher Scientific, Vantaa, Finland) according to the manufacturer's instructions. In parallel, the protein concentration of $50 \mu$ of each sample was assayed with the Bradford method..$^{38}$ The ratio of cholesterol concentration to protein concentration was represented as $\mathrm{mg}$ of cholesterol/mg of cell protein.

\section{Oly labeling, actin labeling, antibodies, and microscopy}

Oly was isolated from fresh fruiting bodies of Pleurotus ostreatus as previously described. ${ }^{18}$ The purity of Oly was checked by polyacrylamide gel electrophoresis. The protein concentration was determined spectrophotometrically using the BCA ${ }^{\text {TM }}$ Protein Assay Reagent (Thermo Scientific Pierce Protein Research Products, Rockford, IL). After isolation, the protein was desalted and kept frozen $\left(-20^{\circ} \mathrm{C}\right)$ in aliquots in $140 \mathrm{mM}$ sodium chloride, $1 \mathrm{mM}$ ethylenediaminetetraacetic acid, $20 \mathrm{mM}$ tris(hydroxymethyl)aminomethane hydrochloride buffer ( $\mathrm{pH}$ 8.0). Rabbit anti-Oly primary antibodies were prepared as previously described. ${ }^{18} \mathrm{~T} 24$ cells grown on coverslips were incubated with $2.5 \mu \mathrm{g} / \mathrm{mL}$ of Oly for 30 minutes at $37^{\circ} \mathrm{C}$. After fixation in $4 \%$ paraformaldehyde, washing with PBS, and blocking with 2\% bovine serum albumin (SigmaAldrich) with $0.2 \%$ sodium azide, (Fluka Chemie, Buch, Switzerland) primary Oly antirabbit antibodies (1:2500) and then Molecular Probes ${ }^{\circledR}$ Alexa Fluor ${ }^{\circledR}$ 555-conjugated secondary antibodies (1:1000) (Invitrogen) were added. Coverslips were washed and mounted in VECTASHIELD ${ }^{\circledR}$ with 4',6-diamidino-2-phenylindole (Vector Laboratories, Burlingame, CA).

Actin labeling was performed in $16.7 \mu \mathrm{g} / \mathrm{mL}$ phalloidin (phalloidin-fluorescein isothiocyanate) (Sigma-Aldrich) in 20\% methanol (Carlo Erba Reagenti, Milan, Italy) in PBS for 30 minutes. For preparations which were labeled for Oly, actin labeling with phalloidin-tetramethylrhodamine isothiocyanate (Sigma-Aldrich) was performed after goat antirabbit secondary antibody incubation and 10 minutes washing in PBS. Afterwards, coverslips were decanted and embedded in VECTASHIELD-4',6-diamidino-2-phenylindole and analyzed in a fluorescence microscope $\left(\mathrm{Axio}^{\mathrm{TM}}\right.$ Imager $\mathrm{Z1}$; Carl Zeiss AG, Oberkochen, Germany).

\section{Phase-contrast and fluorescence image acquisition}

Cells were analyzed in a fluorescence microscope (Axio Imager Z1). Phase-contrast images were taken with $63 \times$-water objective (numerical aperture 0.95 ) and fluorescence images with Plan Apochromat ${ }^{\circledR}$ (63×, oil/numerical aperture 1.4; Carl Zeiss).

\section{Morphometric analysis}

Sampling was performed as follows: cover glass with fixed cells was imaged with water immersion objective $(63 \times)$. Images were taken at every second visual field, second horizontal, and second vertical axis. Afterwards, 20 randomly chosen images were analyzed for each treatment by counting the cells and ICNs per image. Only membrane nanotubes that made contact between neighboring cells were counted as ICNs. The ICN density, ie, the number of ICNs per cell, was calculated as follows: ICN density = (total number of ICNs/ total number of cells). Data are reported as mean \pm standard deviation in tables and text.

\section{Live cell imaging}

T24 cells were cultured on glass bottom dishes (MatTek Corporation, Ashland, MA). Time-lapse imaging was obtained on an LSM 510 (Carl Zeiss) confocal microscope using transmission light (oil objective $63 \times$ ). Initially, images of control were taken (data not shown), then the medium was exchanged with cholesterol-free growth medium treated with $5 \mathrm{mM} \mathrm{m} \beta \mathrm{CD}$ and time sequences collected every 18 seconds for 2 hours.

\section{Results Oly and other raft markers}

Previously, it has been demonstrated that Oly preferentially binds to cholesterol-sphingomyelin membrane nanodomains. ${ }^{19,20}$ Figure 1B shows the binding of Oly to cholesterolsphingomyelin membrane nanodomains throughout the plasma membrane and ICNs in T24 cells. Oly preferentially binds rafts enriched with cholesterol and sphingomyelin molecules. Oly was strongly labeled in some of the T24 cells (Figure 1B), which could be due to differences in the concentration of cholesterol-sphingomyelin rafts. Along the ICNs, there was no binding of caveolin-1 and flotillin-1 raft markers, and very little binding of ganglioside GM1 raft marker (data not shown).

The depletion of cholesterol using $\mathrm{m} \beta C D$ and LPPS revealed reduced binding of Oly to the cell surface (Figure 1D), which demonstrated the disappearance of cholesterol-sphingomyelin membrane nanodomains that Oly binds to in the cell membrane. Finally, due to the relatively low concentration of $\mathrm{m} \beta \mathrm{CD}(1 \mathrm{mM})$ and the short treatment time ( 2 hours), there was neither an effect on the cell morphology nor on the ICN density (Figure 1C and D). 

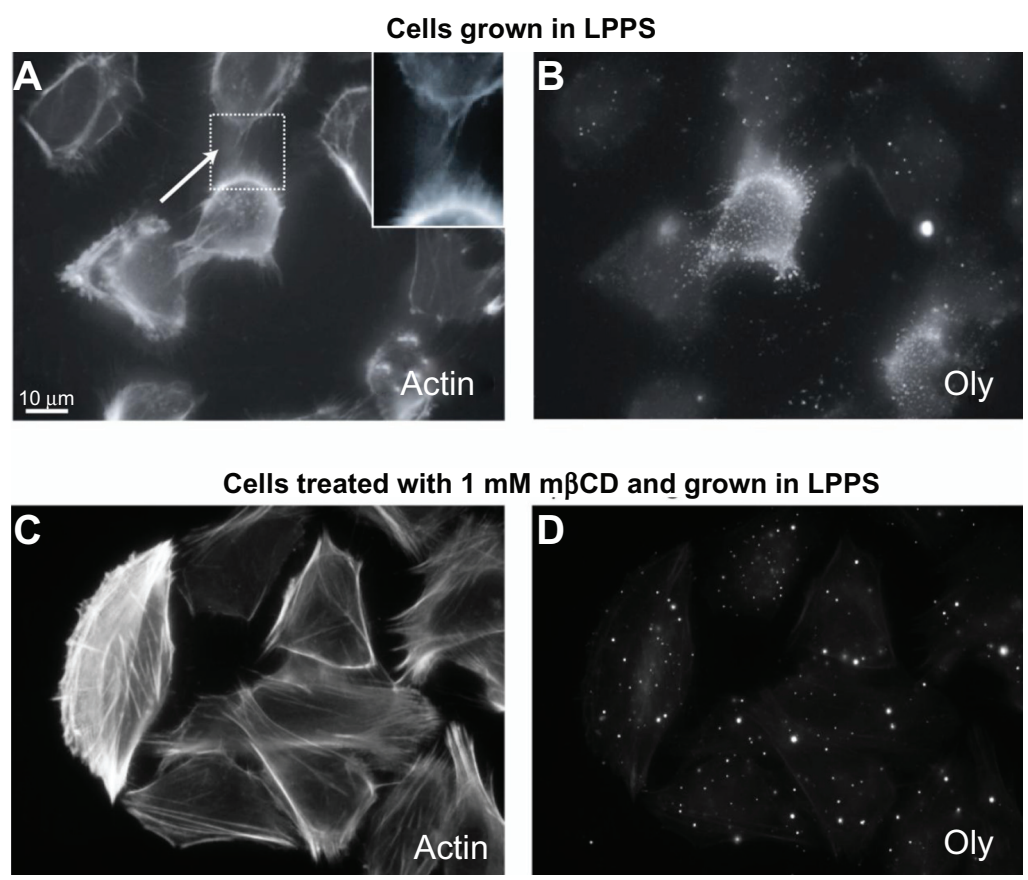

Figure I Ostreolysin marks the presence of cholesterol-sphingomyelin membrane nanodomains along intercellular membrane nanotubes in T24 cells. The labeling of actin filaments highlights the location of intercellular membrane nanotubes (see close up) between T24 (malignant) cells of urothelial origin grown in lipoprotein poor serum medium (A). The addition of ostreolysin reveals specific binding to cholesterol-sphingomyelin membrane nanodomains along the cell surface and along the intercellular membrane nanotubes (B). The growth of T24 cells in lipoprotein poor serum medium treated with I mM methyl- $\beta$-cyclodextrin for 2-hour causes the formation of actin stress fibers (C) as well as the dispersion of cholesterol-sphingomyelin membrane nanodomains (D). Note that the intercellular membrane nanotubes are still present following the application of methyl- $\beta$-cyclodextrin in low concentration (I mM) (C), and that ostreolysin labeling on the cell surface is much reduced (D).

Abbreviations: LPPS, lipoprotein poor serum; $m \beta C D$, methyl- $\beta$-cyclodextrin; Oly, ostreolysin.

\section{$\mathrm{m} \beta C D$ treatment}

CD treatment has been shown to drive the transfer of cholesterol from the outer membrane leaflet of the cell and from fluid areas to $\mathrm{m} \beta \mathrm{CD}$ molecules. ${ }^{39,40}$ In the present study, the depletion of cholesterol from the plasma membrane was verified using the Bligh and Dyer method. It was revealed that the treatment by $\mathrm{m} \beta C D$ reduced the concentration of cholesterol in cell membranes. The relative concentration of cholesterol (mg cholesterol $/ \mathrm{mg}$ protein) was 0.006 in the control experiment, whereas following $\mathrm{m} \beta \mathrm{CD}$ treatment $(4 \mathrm{mM}$ for 2 hours), the relative concentration fell to 0.002 .

Figure 2 shows phase-contrast images of 24 cells grown in LPPS for 2 hours and treated with increasing concentrations of $\mathrm{m} \beta \mathrm{CD}$ (from $2 \mathrm{mM}$ to $20 \mathrm{mM}$ ). Following treatment, cells were more separated, and the extent of cell separation was a function of $\mathrm{m} \beta C D$ concentration. Furthermore, it was revealed that $2 \mathrm{mM} \mathrm{m} \beta C D$ did not have a significant effect on the ICN frequency (Figure 2C). However, above a concentration of $4 \mathrm{mM} \mathrm{m} \beta C D$, the retraction of ICNs became considerable, and the cell edge surface became smoother, demonstrating the retraction of short and long membrane protrusions (Figure 2D-J). These results revealed that the depletion of more cholesterol led to more significant morphologic changes.

\section{Actin and DNA labeling}

The formation of actin stress fibers might be important for cell reshaping and cell contraction. ${ }^{41}$ Following cell growth in LPPS for 4 hours, actin labeling of T24 cells demonstrated the smoothing of the cell surface as well as the formation of actin stress fibers (Figure 3C). The formation of actin stress fibers might facilitate static cell contraction, which could reduce the cell size and smooth out the cell surface. Similar morphologic changes were observed upon $4 \mathrm{mM}$ $\mathrm{m} \beta \mathrm{CD}$ treatment (Figure 3D and E). By adding a DNA label (4',6-diamidino-2-phenylindole), it was observed that the integrity of the cell nuclei, after $\mathrm{m} \beta \mathrm{CD}$ treatment, remained intact (Figure 3F-J).

\section{Morphometric analysis}

To determine the statistical effect of $\mathrm{m} \beta C D$ treatment in T24 cells, the number of cells and the number of ICNs were counted in acquired images. The ICN density was calculated in each image. Summary statistics for the differences in ICN density upon $\mathrm{m} \beta C D$ treatments of different concentrations for 2 hours are shown in Figure 4. It was revealed that the ICN density was significantly $(P<0.05)$ reduced following the application of $4 \mathrm{mM} \mathrm{m} \beta \mathrm{CD}$, and that the average number of ICNs fell dramatically upon an increase in the concentration 

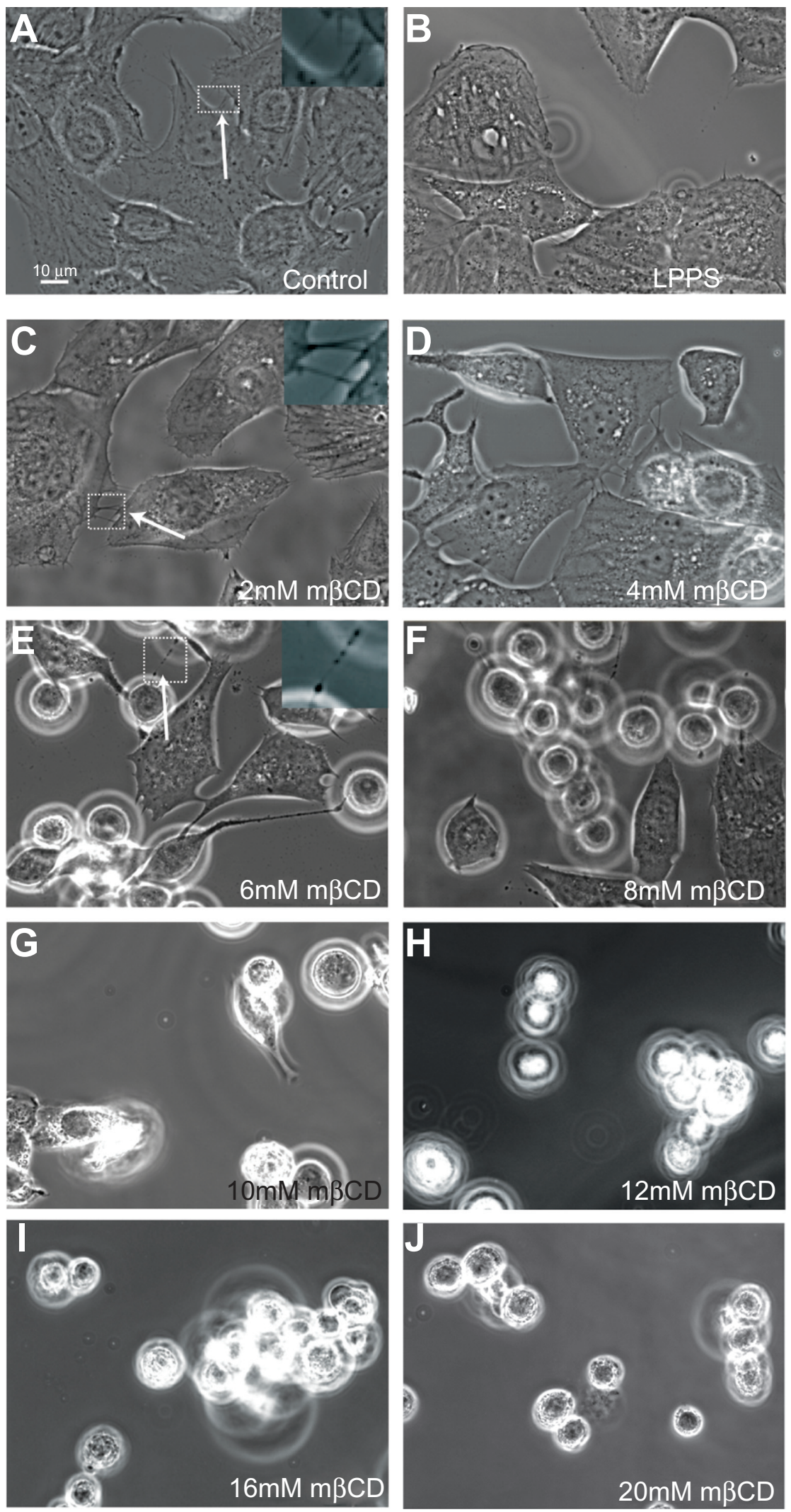

Figure 2 Phase-contrast images of T24 cells revealing the loss of intercellular membrane nanotubes upon increasing concentration of methyl- $\beta$-cyclodextrin treatment. The images show the growth of T24 cells in control (A) and cholesterol-free medium (lipoprotein poor serum) (B), and the morphological changes of T24 cells in lipoprotein poor serum following 2-20 mM methyl- $\beta$-cyclodextrin 2-hour treatment (C-J). The intercellular membrane nanotubes, observed following $4 \mathrm{mM}$ methyl- $\beta$-cyclodextrin treatment, are thinner, longer (C; see arrow), and less frequent than in the control experiment (A; see arrow). The removal of cholesterol from the membrane causes the inward contraction of the cell plasma membrane ( $\mathbf{D}$ and $\mathbf{E})$. The formation of large vesicular dilations along the intercellular membrane nanotube (E; see arrow) could be due to the curvature mismatch between the remaining membrane components and the tube curvature. Above $6 \mathrm{mM}$ methyl- $\beta$-cyclodextrin, the cells become isolated and spherical $(\mathbf{F}-\mathrm{J})$. Abbreviation: $m \beta C D$, methyl- $\beta$-cyclodextrin. 

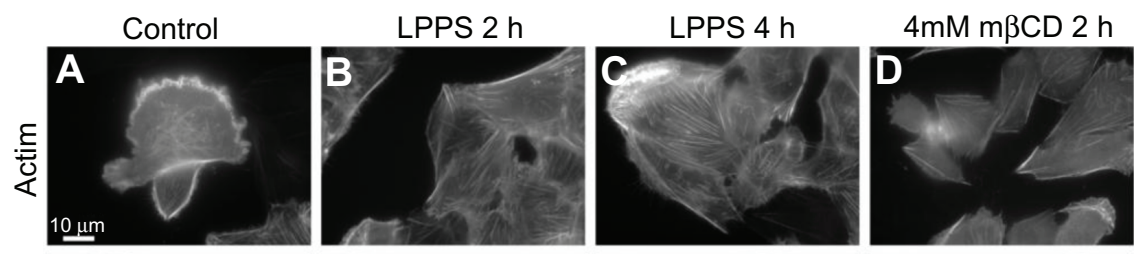

$4 \mathrm{mM} \mathrm{m \beta CD} 4 \mathrm{~h}$
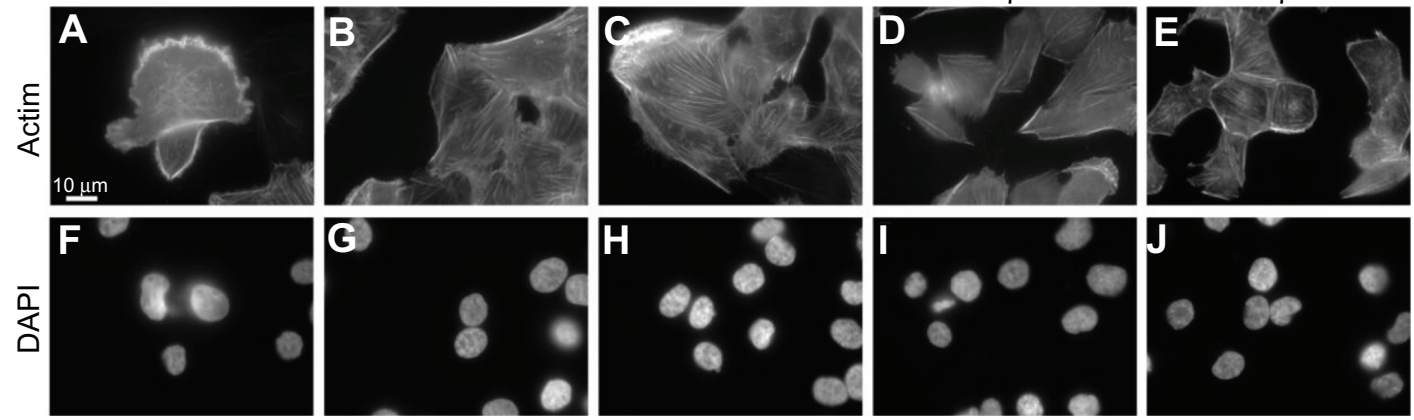

Figure 3 Fluorescence microscope images of T24 cells revealing the integrity of actin filaments and cell nucleus following the growth in cholesterol-free (lipoprotein poor serum) or methyl- $\beta$-cyclodextrin treatments. The actin labeling of T24 cells in the control experiment reveals the cell surface ruffling (A). The growth of T24 cells in lipoprotein poor serum causes the smoothing of the cell surface (retraction of the membrane protrusions) as well as the formation of actin stress fibers, which is observed more after 4-hour (C) than 2-hour (B) treatment. The morphological changes in T24 cells following methyl- $\beta$-cyclodextrin treatment are similar to the changes observed following growth in lipoprotein poor serum (D and E). Using a DNA label (4',6-diamidino-2-phenylindole), the integrity of the cell nuclei in the corresponding lipoprotein poor serum and methyl- $\beta$-cyclodextrin treatments is revealed $(\mathbf{F}-\mathbf{J})$.

Abbreviations: DAPI, 4',6-diamidino-2-phenylindole; h, hours; LPPS, lipoprotein poor serum; m $\beta C D$, methyl- $\beta$-cyclodextrin.

of $\mathrm{m} \beta C D$ from $4 \mathrm{mM}$ to $6 \mathrm{mM}$ (Figure 4 ). This shows that there might be a threshold of cholesterol content in the cell membrane, below which there is a dramatic decline in ICN density.

\section{The coupling between ICN density and cell shaping}

To shed light on the dynamic changes in cell morphology following cholesterol depletion, live cell imaging techniques were employed using confocal laser scan microscopy. The hypothesis was that the disruption of ICNs and the change from a polygonal to a spherical cell shape were mechanically coupled. Using a confocal microscope (LSM 510) and time-lapse imaging, the cell morphologic changes were tracked during treatment of $5 \mathrm{mM} \mathrm{m} \beta \mathrm{CD}$. Figure 5A shows that ICNs are disrupted somewhere in the middle, and then retracted towards the parent cell membrane. Furthermore, the disconnection of ICNs caused the abrupt cell separation and the rounding of a T24 cell (Figure 5).

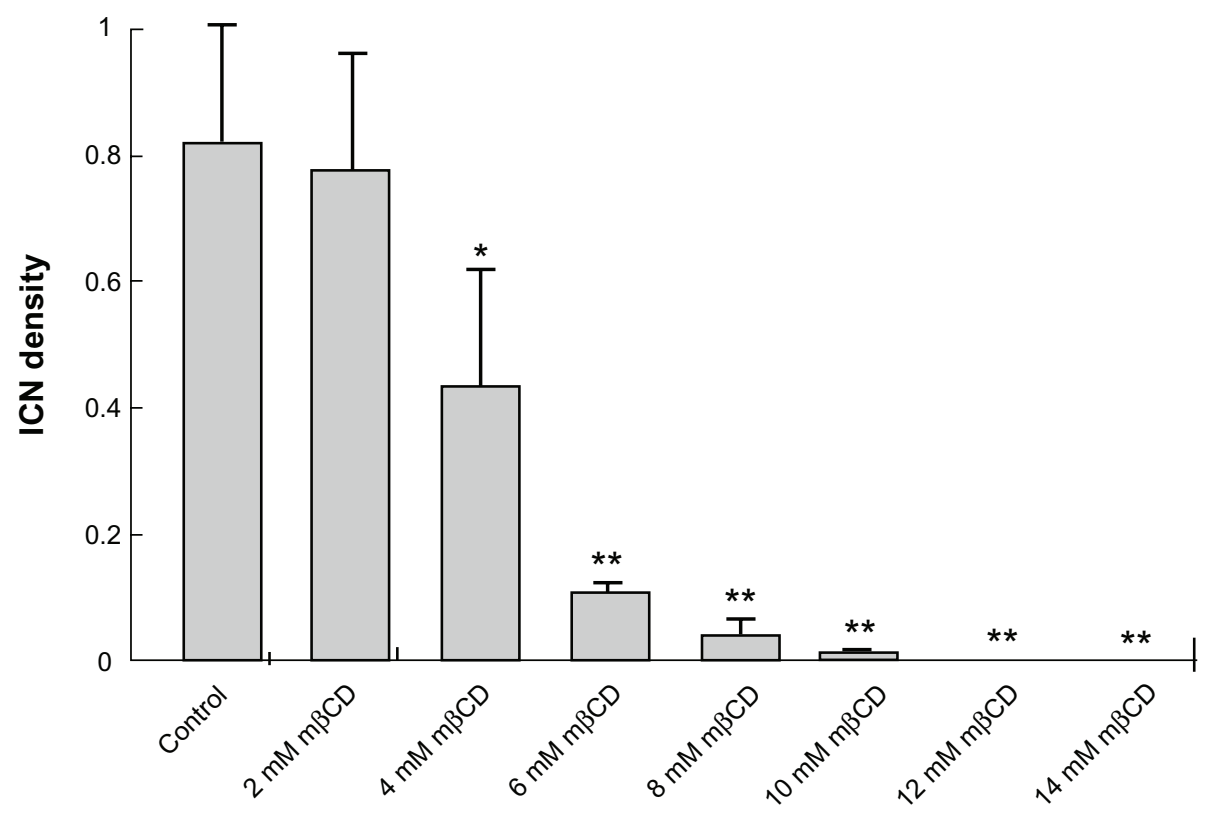

Figure 4 Summary statistics of changes in intercellular membrane nanotube density among T24 cells grown in different growth mediums and following different methyl- $\beta$ cyclodextrin concentration treatments. The statistical differences are calculated with respect to the control case. Note that the reduction in intercellular membrane nanotube density becomes significant from $4 \mathrm{mM}$ methyl- $\beta$-cyclodextrin 2-hour treatment and highly significant above this concentration.

Note: Data are mean \pm standard deviation; $* P<0.05$; $* * p<0.01$.

Abbreviations: ICN, intercellular membrane nanotube; $m \beta C D$, methyl- $\beta$-cyclodextrin. 
A

$1 \mathrm{~h} 3 \mathrm{~m} 18 \mathrm{~s}$

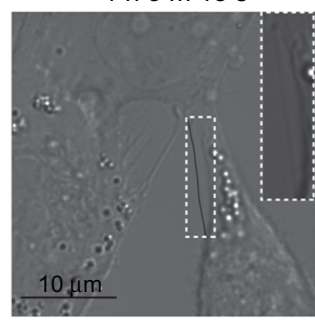

B

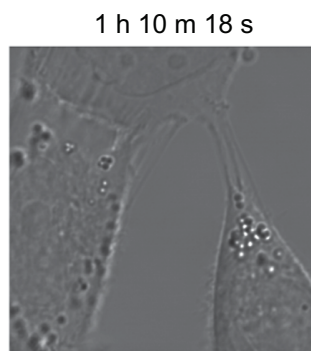

Rupturing of an ICN

$1 \mathrm{~h} 4 \mathrm{~m} 36 \mathrm{~s}$

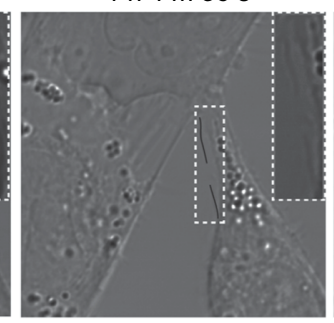

$1 \mathrm{~h} 5 \mathrm{~m} 12 \mathrm{~s}$

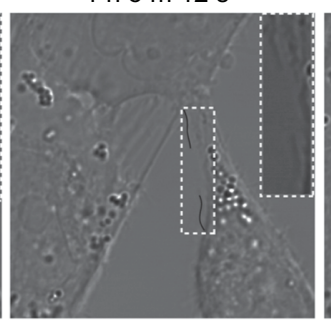

Cell seperation

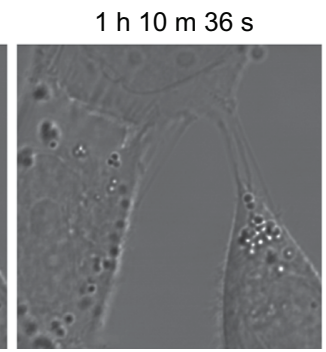

$1 \mathrm{~h} 10 \mathrm{~m} 54 \mathrm{~s}$

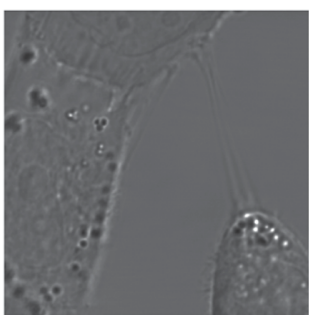

$1 \mathrm{~h} 6 \mathrm{~m} 54 \mathrm{~s}$

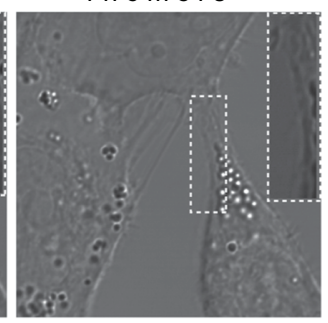

$1 \mathrm{~h} 11 \mathrm{~m} 12 \mathrm{~s}$

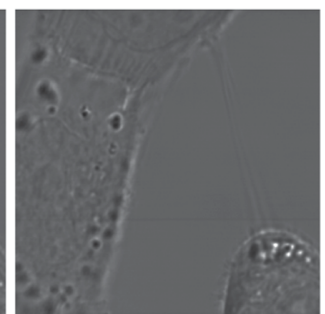

Figure 5 Phase contrast images demonstrating the coupling between intercellular membrane nanotube disconnection and cell separation. At time zero, the T24 cells were treated with $5 \mathrm{mM}$ methyl- $\beta$-cyclodextrin. The disruption of intercellular membrane nanotubes was observed using time-lapse confocal microscopy for the indicated times (A). Note that the intercellular membrane nanotube (see inset) is disrupted midway and then retracted. The disconnection of intercellular membrane nanotubes, possibly due to stretching, causes the abrupt cell separation, accompanied by morphological alterations from a polygonal to a spherical cell shape (B).

Note: Scale bar is $10 \mu \mathrm{m}$.

Abbreviations: $h$, hours; ICN, intercellular membrane nanotube; $\mathrm{m}$, minutes; $\mathrm{s}$, seconds.

\section{Possible mechanisms for $\mathrm{m} \beta C D$-induced morphological changes of T24 cells}

There are three possible mechanisms responsible for the observed reduction in the ICN density during $\mathrm{m} \beta C D$ treatment. The first mechanism is that the "gaps" created in the cell membrane, due to cholesterol depletion from the external leaflet and fluid areas, were filled by the surrounding membrane, thereby causing the contraction of the cell membrane and the partial retraction of membrane protrusions. The second possible mechanism is based on the positive spontaneous curvature of a cholesterolphospholipid complex (nanodomain) (Figure 6). This spontaneous curvature could be due to an area difference between the two membrane leaflets, assuming that more cholesterol molecules might reside in the outer than in the inner membrane leaflet (Figure 6A), or due to a spontaneous curvature of the cholesterol-phospholipid complex (Figure 6B). The plasma membrane of the
A

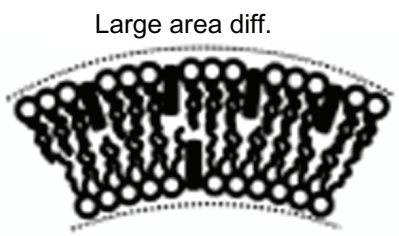

B

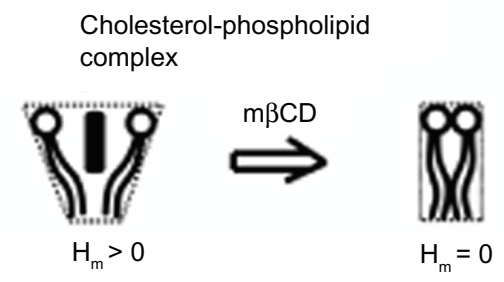

Small area diff.
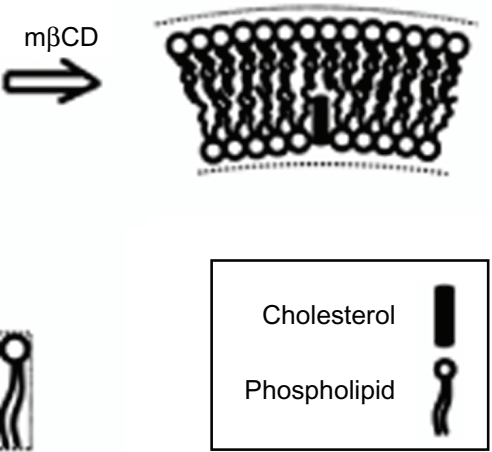

Figure 6 Schematic diagram for the possible effects of methyl- $\beta$-cyclodextrin on the curvature of cholesterol-enriched nanodomains. A larger cholesterol content in the outer than in the inner membrane leaflet would increase the local curvature of a membrane region (A). Note that cholesterol depletion from the outer membrane leaflet is predicted to reduce the area difference between the two membrane leaflets. Due to cholesterol short sterol backbone, a cholesterol-phospholipid complex might have a positive spontaneous curvature, which is reduced upon methyl- $\beta$-cyclodextrin treatment (B).

Abbreviations: $H_{m}$, spontaneous mean curvature of the nanodomain; $m \beta C D$, methyl- $\beta$-cyclodextrin. 
A

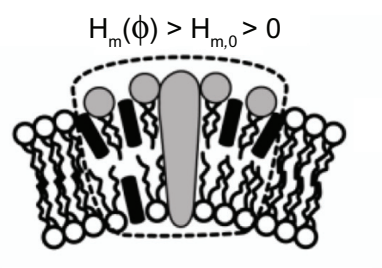

Cholesterol-sphingomyelin nanodomain raft
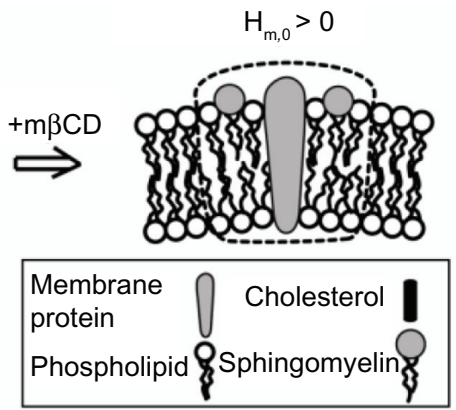

B
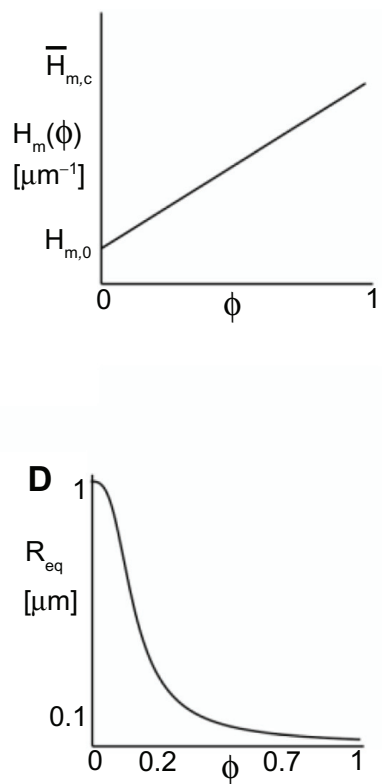

C

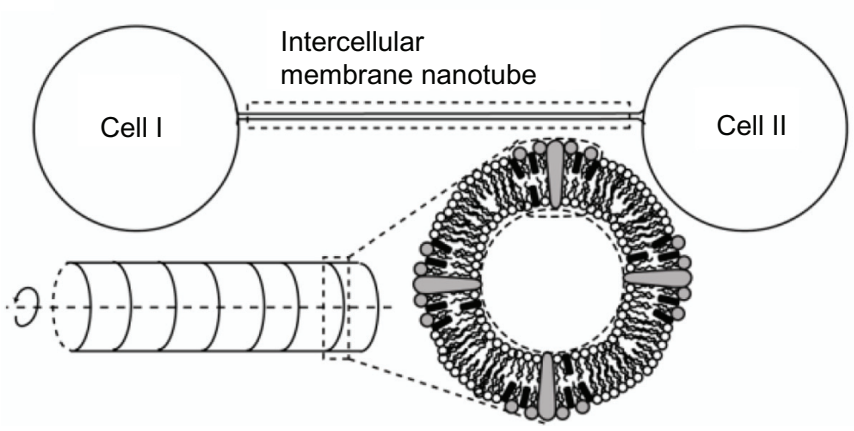

Figure 7 Schematic diagram for the possible model explaining the effects of cholesterol depletion on the intercellular membrane nanotube geometry. A cholesterolsphingomyelin membrane nanodomain is assumed to have a positive spontaneous curvature, which is reduced following cholesterol depletion (A). A linear relationship is assumed between the spontaneous curvature of the nanodomain and its cholesterol content (B). The same relationship is assumed for the spontaneous membrane curvature deviator. The intercellular membrane nanotube is modeled as an axisymmetric tubular structure, which is composed of cholesterol-sphingomyelin membrane nanodomain of positive spontaneous curvature (C). The equilibrium radius is obtained by the employed linear stability analysis and plotted as a function of cholesterol content (D). Note that cholesterol depletion $(\phi=0)$ favors larger intercellular membrane nanotube radii. The parameter values used are as follows: spontaneous mean $\left(H_{m, 0}\right)$ and deviatoric $\left(D_{m, 0}\right)$ curvature of the cholesterol-free nanodomain $=I \mu \mathrm{m}^{-1}$, spontaneous mean $\left(\bar{H}_{m, c}\right)$ and deviatoric $\left(\bar{D}_{m, c}\right)$ curvature of the nanodomain $=25 \mu \mathrm{m}^{-1}, \sigma=0.00 \mathrm{I} g \mathrm{~s}^{-2}$, and membrane bending rigidity $\kappa=500 \mathrm{k}_{\mathrm{B}} \mathrm{T}$, where $\mathrm{k}_{\mathrm{B}}$ is the Boltzmann constant and $\mathrm{T}$ is temperature.

Abbreviations: $\phi$, cholesterol content; $\mathrm{H}_{\mathrm{m}}(\phi)$, spontaneous mean curvature of the nanodomain; $\mathrm{H}_{\mathrm{m}, 0}$, spontaneous mean curvature of the cholesterol-free nanodomain; $\mathrm{m} \beta C D$, methyl- $\beta$-cyclodextrin; $R_{\text {eq }}$, equilibrium radius.

human erythrocyte contains in the outer membrane leaflet $65 \%-75 \%$ of the phosphatidylcholine and $>85 \%$ of the sphingomyelin, while in the inner membrane leaflet there is $80 \%-85 \%$ of the phosphatidylethanolamine and $>96 \%$ of the phosphatidylserine. ${ }^{42}$ Since the outer membrane leaflet is enriched in sphingomyelin, the preferred interaction of cholesterol with sphingomyelin may justify the assumption of cholesterol residing more in the outer than in the inner membrane leaflet of the ICN. The third possible (complementary) mechanism is that cell separation stretches the ICNs, until the ICNs are disrupted in the middle or detached from the parent cell membrane.

To minimize the bending energy costs during the formation of a thin tubular structure, cholesterol molecules could accumulate in the outer membrane leaflet. Therefore, the depletion of cholesterol from the outer membrane leaflet would increase the mismatch between the tube curvature and the spontaneous curvature of the remaining membrane nanodomains.

\section{The model of ICN stability and theoretical predictions}

Phase-contrast images of T24 cells grown in LPPS and $\mathrm{m} \beta C D$ for 2 hours revealed that during cell separation the ICNs became thinner and longer and, eventually, disrupted (Figures 2 and 5). The aim of the present model was to elucidate the possible underlying mechanisms responsible for the disruption of ICNs. The computational model was an extension of a previous model. ${ }^{10}$ The model included only the ICN isolated, ie, it excluded the membrane of the parent cells (Figure 7C). The ICN was modeled as an axisymmetric shape of cylindrical symmetry. For the sake of simplicity, the membrane was composed of only one component (nanodomain). The component was a cholesterol-sphingomyelin membrane nanodomain, consisting of cholesterol, sphingomyelin, protein, and other lipid molecules (Figure 7A). The spontaneous curvature of the nanodomain was assumed to be positive, which was based on either nonzero area difference between the two 
membrane leaflets (Figure 6A) or the intrinsic curvature of the cholesterol-phospholipid complex (Figure 6B). The underlying assumption of the model was the asymmetry in the cholesterol content between the inner and outer membrane leaflets along the axisymmetric tubular structure, such that there were more cholesterol molecules in the outer than in the inner membrane leaflet of the ICN (Figure 6), influencing the nanodomain intrinsic (spontaneous) curvature. The sign of the membrane curvature was defined with respect to the outer membrane leaflet. The membrane bilayer was modeled as a single entity.

The membrane free (bending) energy is considered within continuum approach in the form: ${ }^{10,43}$

$$
\mathrm{E}=\int\left(\kappa\left(\mathrm{H}-\mathrm{H}_{\mathrm{m}}(\phi)\right)^{2}+\kappa\left(\mathrm{D}-\mathrm{D}_{\mathrm{m}}(\phi)\right)^{2}+\sigma\right) \mathrm{dA}
$$

where $\kappa$ is the membrane bending rigidity, $\phi$ is the relative density of cholesterol molecules in the outer membrane leaflet, $\mathrm{H}$ is the mean membrane curvature, $\mathrm{D}$ is the local membrane curvature deviator, $\mathrm{H}_{\mathrm{m}}(\phi)$ is the intrinsic (spontaneous) mean curvature of the nanodomain, $\mathrm{D}_{\mathrm{m}}(\phi)$ is the intrinsic (spontaneous) membrane curvature deviator of the nanodomain, $\sigma$ is the Lagrange multiplier, and $\mathrm{dA}$ is the infinitesimal membrane area element. The integral is taken over the surface of the axisymmetric tube. The mean membrane curvature takes into account the two principal curvatures of the axisymmetric cylindrical structure. The difference between the membrane curvature and the membrane intrinsic (spontaneous) curvature determines the energy cost for bending the membrane away from its favorable curvature. ${ }^{43}$

The intrinsic (spontaneous) curvatures of the membrane nanodomain depend on the relative density of cholesterol molecules in the outer lipid layer, as follows:

$$
\begin{aligned}
& \mathrm{H}_{\mathrm{m}}(\phi)=\mathrm{H}_{\mathrm{m}, 0}+\overline{\mathrm{H}}_{\mathrm{m}, \mathrm{c}} \phi \\
& \mathrm{D}_{\mathrm{m}}(\phi)=\mathrm{D}_{\mathrm{m}, 0}+\overline{\mathrm{D}}_{\mathrm{m}, \mathrm{c}} \phi
\end{aligned}
$$

where $\mathrm{H}_{\mathrm{m}, 0}$ and $\mathrm{D}_{\mathrm{m}, 0}$ are the intrinsic (spontaneous) mean and deviatoric curvature of the cholesterol-free nanodomain, respectively, and $\mathrm{H}_{\mathrm{m}, \mathrm{c}}$ and $\mathrm{D}_{\mathrm{m}, \mathrm{c}}$ are the intrinsic (spontaneous) mean and deviatoric curvature of the cholesterol-replenished (maximum concentration) nanodomain, respectively.

The equation of motion of the membrane height deflection along the cylinder main symmetry axis is given by:

$$
\varphi \frac{\delta \mathrm{h}(\mathrm{z})}{\delta \mathrm{t}}=-\frac{\partial \mathrm{E}}{\partial \mathrm{h}(\mathrm{z})}
$$

where $\varphi$ is the friction coefficient describing the drag of the fluid surrounding the membrane, $t$ is time, and $\mathrm{E}$ is redefined as the membrane free energy per unit area. The system is investigated in the limit of small membrane deformation from the uniform radius of the ICN. By linearization of Equation (3), the following equation was obtained:

$$
\varphi \frac{\delta \mathrm{h}(\mathrm{z})}{\delta \mathrm{t}}=\int\left(\mathrm{U}+\mathrm{L}(\mathrm{h}) \delta+\mathrm{O}\left(\delta^{2}\right)\right) \mathrm{dA},
$$

where the function $\mathrm{L}(\mathrm{h})$ describes the force in the limit of small and linear membrane undulations. The integral sign is for the calculation of the average force density. The force acting on the membrane in the equilibrium state is described by $\mathrm{U}$. The equilibrium radius $\mathrm{R}_{\mathrm{eq}}$ is obtained by assuming that the undulation $\mathrm{U}$ equals zero, which yields:

$$
\mathrm{R}_{\mathrm{eq}}=\frac{\sqrt{\kappa}}{\sqrt{2} \sqrt{\sigma+\left(\mathrm{H}_{\mathrm{m}}(\phi)^{2}+\mathrm{D}_{\mathrm{m}}(\phi)^{2}\right) \kappa \phi^{2}}}
$$

It is important to note that the axisymmetric model of the ICN is stable even without anisotropy, since it is not considered as a membrane protrusion.

An aim of this study was to analyze the effects of changes in the relative density of cholesterol on the equilibrium radius of the tube. Figure 7D shows the equilibrium radius as a function of the relative area density of cholesterol. The equilibrium radius remains low at $0.15 \mu \mathrm{m}$ for a relative density of 0.3 . When the relative density is reduced below 0.2 , the equilibrium radius is increased rapidly. A plausible range of cholesterol content in the plasma membrane is between $20 \%-30 \%$. According to calculations, the cholesterol content might be a critical parameter, having a role not only in the stability of membrane nanodomains but also affecting the radius of the highly curved structure. The complete depletion of cholesterol $(\phi=0)$ from the outer leaflet of the tubular membrane is predicted to considerably increase the curvature mismatch between the spontaneous curvature of the remaining membrane components and the tube curvature. To reduce the curvature mismatch, vesicular dilations could be formed during the retraction of the ICN (Figure 2E).

\section{Discussion}

This study demonstrates the presence of cholesterolsphingomyelin membrane nanodomains along ICNs, which was revealed by the addition of membrane raft marker Oly (Figure 1). However, the ICNs were negative for the binding of other membrane raft makers such as flotilin-1, caveolin-1, 
and ganglioside GM1. The removal of cholesterol from the cell membrane by the application of $\mathrm{m} \beta C D$ led to the smoothing of the cell surface, the separation of cells, the stretching of ICNs, and the retraction of disconnected ICNs (Figure 2). Following cholesterol depletion, the labeling of actin filaments revealed the formation of actin stress fibers and the reorganization of the actin cytoskeleton (Figure 3 ). Statistical analysis revealed significant $(P<0.01)$ reductions in the density of ICNs upon exposure to high concentrations of $\mathrm{m} \beta C D$ (Figure 4). The change of cell shape from polygonal to spherical would increase the distance between neighboring cells, stretching the connected ICNs. A computational model was constructed to investigate the contributions of changes in the spontaneous (intrinsic) curvature of cholesterol-sphingomyelin membrane nanodomains to the shape and stability of ICNs (Figures 6 and 7). It was theoretically predicted that the depletion of cholesterol from the outer membrane leaflet would favor a larger radius of the tubular structure. Actually, the reduction in the ICN radius was observed (Figure 5), which increases the curvature mismatch between the tube curvature and the spontaneous curvatures $\left(\mathrm{H}_{\mathrm{m}}(\phi)\right.$ and $\left.\mathrm{D}_{\mathrm{m}}(\phi)\right)$ of the remaining membrane components. This curvature mismatch could be reduced by the formation of large vesicular dilations along the ICN (Figure 2E), and by the detachment of ICNs from the parent plasma membranes (Figure 5A).

CDs have been mainly used for the rapid extraction of cholesterol molecules from cell membranes, ${ }^{39,40}$ while the release of phospholipids was only minimal. ${ }^{32,33}$ Previous studies have shown that the depletion of cholesterol by CDs might lead to a reduction in endocytosis and membrane invagination. ${ }^{34-36}$ Yet, the underlying mechanism is probably different from the one in the present model, since membrane invaginations are enriched in cholesterol-based caveolin nanodomains, which do not contain sphingomyelin molecules. The underlying assumption of the present model is that the cholesterol-sphingomyelin complex has a positive spontaneous curvature, whereas, cholesterol-based caveolin nanodomains may have a negative spontaneous curvature.

The depletion of cholesterol from the outer membrane leaflet may reduce the area difference between the two membrane leaflets. As a result, the plasma membrane would favor more planar-like conformations, explaining the smoothing of the cell surface and the retraction of disconnected ICNs (Figure 2 and Figure 5A). Since it has been recently shown that cholesterol-sphingomyelin nanodomains were essential for microtubule-based protrusion growth, ${ }^{44}$ it could be also possible that the stability of the ICN depends on the binding of microtubules to the ICN membrane through cholesterol-based membrane nanodomains.

According to the theory of isotropic membrane elasticity, ${ }^{45,46}$ the cell tubular membrane protrusion cannot be stabilized by isotropic membrane components alone, ${ }^{43}$ but requires the accumulation of anisotropic membrane nanodomains. ${ }^{6,8,10,47,48}$ Previous theoretical studies provide insight into the stability of the tubular membrane protrusion following the disassembly of the inner rod cytoskeleton, ${ }^{4}$ or into the stability of actin-free filopodia. ${ }^{10,49}$ Accordingly, it is possible that the accumulation of cholesterol-sphingomyelin membrane nanodomains of anisotropic spontaneous curvature may contribute to the tube stability by overcoming the decrease in configurational entropy during the process of lateral sorting of the nanodomains. ${ }^{8,6,10,48-50}$ Therefore, the loss of cholesterol-sphingomyelin rafts that may be connected to reduced intrinsic anisotropy of the tube membrane might cause dynamic instability leading to the retraction of disconnected ICNs.

In a previous study, it has been shown that cholesterolsphingomyelin membrane domains protrude out of the membrane surface, ${ }^{51}$ thereby increasing the lateral tension between the protruding ordered nanodomain and the neighboring disordered nanodomains. The increased membrane tension can be reduced by cholesterol molecules bridging the membrane height difference between the ordered and disordered nanodomains. It is also possible that the high curvature along ICNs would introduce additional membrane tension. Consequently, the cholesterol content in cholesterolsphingomyelin membrane nanodomains along ICNs could be greater than in similar nanodomains on the cell (spherical) plasma membrane.

In conclusion, the present experimental and theoretical results suggest that the transfer of cholesterol from the outer membrane leaflet of cell membranes of malignant T24 cells to CDs molecules causes the smoothing of the cell surface accompanied by the retraction of ICNs. A future study will investigate whether these morphologic alterations are due to the amounts of membrane lipids being depleted or due to the change in the spontaneous curvature of the cholesterol-free membrane regions. The possibility of different membrane nanodomain composition organized by the high cylindrical curvature of ICNs could be investigated using cholesterol/ sphingomyelin probe Oly.

As the ICNs are supposed to represent an important communication system enabling metastatic cancer cells to explore their surroundings, ${ }^{7}$ their reduction by cholesterol removal might represent a new target for anticancer therapy. 
The exploitation of ICNs for the movement of pathogens (eg, viral particles such as human immunodeficiency virus $)^{52}$ on top of the membrane nanotubes from infected to noninfected cells might be dependent on cholesterol-based membrane nanodomains. The contributions of hopanoids (the bacterial equivalent of cholesterol) to the stability of membrane nanotubes that confer antibiotic resistance ${ }^{53}$ could be explored in a future study. The present results regarding the functional link between the membrane composition and the stability of the ICN may shed light on the intercellular communication between neighboring cells.

\section{Acknowledgments}

This work was supported by ARRS grants J3-9219-0381 and P2-0232-1538.

\section{Disclosure}

The authors report no conflicts of interest in this work.

\section{References}

1. Gerdes HH, Carvalho RN. Intercellular transfer mediated by tunneling nanotubes. Curr Opin Cell Biol. 2008;20(4):470-475.

2. Gürke S, Barroso JF, Gerdes HH. The art of cellular communication: tunneling nanotubes bridge the divide. Histochem Cell Biol. 2008; 129(5):539-550

3. Sherer NM, Mothes W. Cytonemes and tunneling nanotubules in cell-cell communication and viral pathogenesis. Trends Cell Biol. 2008;18(9):414-420

4. Veranič P, Lokar M, Schutz GJ, et al. Different types of cell-tocell connections mediated by nanotubular structures. Biophys $J$. 2008;95(9):4416-4425.

5. Lokar M, Perutková S, Kralj-Iglič V, Iglič A, Veranič P. Membrane nanotubes in urothelial cell line T24. In: Liu L, Iglic A, editors. Advances in Planar Lipid Bilayers and Liposomes, Volume 10. Burlington, VT: Academic Press; 2009:65-94.

6. Hurtig J, Chiu DT, Onfelt B. Intercellular nanotubes: insights from imaging studies and beyond. Wiley Interdiscip Rev Nanomed Nanobiotechnol. 2010;2(3):260-276.

7. Kabaso D, Lokar M, Kralj-Iglič V, Veranič P, Iglič A. Temperature and cholera toxin B are factors that influence formation of membrane nanotubes in RT4 and T24 urothelial cancer cell lines. Int J Nanomedicine. 2011;6:495-509.

8. Iglič A, Hägerstrand H, Veranič P, Plemenitaš A, Kralj-Iglič V. Curvature-induced accumulation of anisotropic membrane components and raft formation in cylindrical membrane protrusions. J Theor Biol. 2006;240(3):368-373.

9. Kabaso D, Shlomovitz R, Schloen K, Stradal T, Gov NS. Theoretical model for cellular shapes driven by protrusive and adhesive forces. PLoS Comput Biol. 2011;7(5):e1001127.

10. Kabaso D, Bobrovska N, Góźdź W, et al. On the role of membrane anisotropy and BAR proteins in the stability of tubular membrane structures. J Biomech. 2012;45(2):231-238.

11. Chowdhury HH, Rebolj K, Kreft M, Zorec R, Maček P, Sepčić K. Lysophospholipids prevent binding of a cytolytic protein ostreolysin to cholesterol-enriched membrane domains. Toxicon. 2008;51(8): $1345-1356$.

12. Resnik N, Sepčić K, Plemenitaš A, Windoffer R, Leube R, Veranič $P$. Desmosome assembly and cell-cell adhesion are membrane raftdependent processes. J Biol Chem. 2011;286(2):1499-1507.
13. Ohno-Iwashita Y, Shimada Y, Waheed AA, et al. Perfringolysin O, a cholesterol-binding cytolysin, as a probe for lipid rafts. Anaerobe. 2004; 10(2):125-134.

14. Ishitsuka R, Sato SB, Kobayashi T. Imaging lipid rafts. J Biochem. 2005;137(3):249-254.

15. Hullin-Matsuda F, Kobayashi T. Monitoring the distribution and dynamics of signaling microdomains in living cells with lipid-specific probes. Cell Mol Life Sci. 2007;64(19-20):2492-2504.

16. Chinnapen DJ, Chinnapen H, Saslowsky D, Lencer WI. Rafting with cholera toxin: endocytosis and trafficking from plasma membrane to ER. FEMS Microbiol Lett. 2007;266(2):129-137.

17. Sakaguchi O, Shimada H, Yokota K. Proceedings: purification and characteristics of hemolytic toxin from Aspergillus fumigatus. Jpn J Med Sci Biol. 1975;28(5-6):328-331.

18. Berne S, Križaj I, Pohleven F, Turk T, Maček P, Sepčić K. Pleurotus and Agrocybe hemolysins, new proteins hypothetically involved in fungal fruiting. Biochim Biophys Acta. 2002;1570(3): $153-159$.

19. Sepčić K, Berne S, Rebolj K, et al. Ostreolysin, a pore-forming protein from the oyster mushroom, interacts specifically with membrane cholesterol-rich lipid domains. FEBS Lett. 2004;575(1-3):81-85.

20. Rebolj K, Ulrih NP, Maček P, Sepčić K. Steroid structural requirements for interaction of ostreolysin, a lipid-raft binding cytolysin, with lipid monolayers and bilayers. Biochim Biophys Acta. 2006;1758(10): 1662-1670.

21. Berne S, Lah L, Sepčić K. Aegerolysins: structure, function, and putative biological role. Protein Sci. 2009;18(4):694-706.

22. London $E$. Insights into lipid raft structure and formation from experiments in model membranes. Curr Opin Struct Biol. 2002;12(4):480-486.

23. Simons K, Ikonen E. Functional rafts in cell membranes. Nature. 1997;387(6633):569-572.

24. Edidin M. The state of lipid rafts: from model membranes to cells. Annu Rev Biophys Biomol Struct. 2003;32:257-283.

25. Chen Z, Rand RP. The influence of cholesterol on phospholipid membrane curvature and bending elasticity. Biophys J. 1997;73(1):267-276.

26. Churchward MA, Rogasevskaia T, Brandman DM, et al. Specific lipids supply critical negative spontaneous curvature - an essential component of native $\mathrm{Ca}^{2+}$-triggered membrane fusion. Biophys $J$. 2008;94(10):3976-3986.

27. de Almeida RF, Fedorov A, Prieto M. Sphingomyelin/phosphatidylcholine/cholesterol phase diagram: boundaries and composition of lipid rafts. Biophys J. 2003;85(4):2406-2416.

28. Baumgart T, Hess ST, Webb WW. Imaging coexisting fluid domains in biomembrane models coupling curvature and line tension. Nature. 2003;425(6960):821-824.

29. McConnell HM, Vrljic M. Liquid-liquid immiscibility in membranes. Annu Rev Biophys Biomol Struct. 2003;32:469-492.

30. Loftsson T, Duchene D. Cyclodextrins and their pharmaceutical applications. Int J Pharm. 2007;392(1-2):1-11.

31. Stella VJ, He Q. Cyclodextrins. Toxicol Pathol. 2008;36(1):30-42.

32. Kilsdonk EP, Yancey PG, Stoudt GW, et al. Cellular cholesterol efflux mediated by cyclodextrins. J Biol Chem. 1995;270(29): 17250-17256.

33. Fukasawa M, Nishijima M, Itabe H, Takano T, Hanada K. Reduction of sphingomyelin level without accumulation of ceramide in Chinese hamster ovary cells affects detergent-resistant membrane domains and enhances cellular cholesterol efflux to methyl-beta-cyclodextrin. J Biol Chem. 2000;275(44):34028-34034.

34. Grimmer S, van Deurs B, Sandvig K. Membrane ruffling and macropinocytosis in A431 cells require cholesterol. J Cell Sci. 2002;115(Pt 14): 2953-2962.

35. Fittipaldi A, Ferrari A, Zoppe M, et al. Cell membrane lipid rafts mediate caveolar endocytosis of HIV-1 Tat fusion proteins. J Biol Chem. 2003;278(36):34141-34149.

36. Romer W, Berland L, Chambon V, et al. Shiga toxin induces tubular membrane invaginations for its uptake into cells. Nature. 2007;450(7170):670-675. 
37. Bligh EG, Dyer WJ. A rapid method of total lipid extraction and purification. Can J Biochem Physiol. 1959;37(8):911-917.

38. Bradford MM. Rapid and sensitive method for the quantitation of microgram quantities of protein utilizing the principle of protein-dye binding. Anal Biochem. 1976;72:248-254.

39. Yancey PG, Rodrigueza WV, Kilsdonk EP, et al. Cellular cholesterol efflux mediated by cyclodextrins. Demonstration of kinetic pools and mechanism of efflux. J Biol Chem. 1996;271(27):16026-16034.

40. Ohvo-Rekila H, Ramstedt B, Leppimaki P, Slotte JP. Cholesterol interactions with phospholipids in membranes. Prog Lipid Res. 2002; 41(1):66-97.

41. Pellegrin S, Mellor H. Actin stress fibres. J Cell Sci. 2007;120(Pt 20): 3491-3499.

42. Zachowski A. Phospholipids in animal eukaryotic membranes: transverse asymmetry and movement. Biochem J. 1993;294(Pt 1):1-14.

43. Iglič A, Babnik B, Gimsa U, Kralj-Iglič V. On the role of membrane anisotropy in the beading transition of undulated tubular membrane structures. J Phys A Math Gen. 2005;38(40):8527-8536.

44. Schwan C, Nolke T, Kruppke AS, Schubert DM, Lang AE, Aktories K. Cholesterol- and sphingolipid-rich microdomains are essential for microtubule-based membrane protrusions induced by Clostridium difficile transferase (CDT). J Biol Chem. 2011;286(33):29356-29365.

45. Helfrich W. Elastic properties of lipid bilayers: theory and possible experiments. Z Naturforsch C. 1973;28(11):693-703.
46. Kabaso D, Gongadze E, Elter P, van Rienen KU, Gimsa J, Iglič A. Attachment of rod-like (BAR) and membranes shape. Mini Rev Med Chem. 2011;11:272-282.

47. Miao L, Fourcade B, Rao M, Wortis M, Zia RK. Equilibrium budding and vesiculation in the curvature model of fluid lipid vesicles. Phys Rev E. 1991;43(12):6843-6856.

48. Iglič A, Slivnik T, Kralj-Iglič V. Elastic properties of biological membranes influenced by attached proteins. J Biomech. 2007;40(11): 2492-2500.

49. Yang C, Hoelzle M, Disanza A, Scita G, Svitkina T. Coordination of membrane and actin cytoskeleton dynamics during filopodia protrusion. PLoS One. 2009;4(5):e5678.

50. Gongadze E, Kabaso D, Bauer S, et al. Adhesion of osteoblasts to nanorough implant titanium surface. Int J Nanomedicine. 2011;6: 1801-1816.

51. Lawrence JC, Saslowsky DE, Edwardson JM, Henderson RM. Realtime analysis of the effects of cholesterol on lipid raft behavior using atomic force microscopy. Biophys J. 2003;84(3):1827-1832.

52. Sowinski S, Jolly C, Berninghausen $\mathrm{O}$, et al. Membrane nanotubes physically connect $\mathrm{T}$ cells over long distances presenting a novel route for HIV-1 transmission. Nat Cell Biol. 2008;10(2):211-219.

53. Dubey GP, Ben-Yehuda S. Intercellular nanotubes mediate bacterial communication. Cell. 2011;144(4):590-600.
International Journal of Nanomedicine

\section{Publish your work in this journal}

The International Journal of Nanomedicine is an international, peerreviewed journal focusing on the application of nanotechnology in diagnostics, therapeutics, and drug delivery systems throughout the biomedical field. This journal is indexed on PubMed Central, MedLine, CAS, SciSearch $\AA$, Current Contents ${ }^{\circledR} /$ Clinical Medicine,

\section{Dovepress}

Journal Citation Reports/Science Edition, EMBase, Scopus and the Elsevier Bibliographic databases. The manuscript management system is completely online and includes a very quick and fair peer-review system, which is all easy to use. Visit http://www.dovepress.com/ testimonials.php to read real quotes from published authors. 